\title{
Prüfung eines Stromentnahmesystems
}

\author{
Manfred Deutzer
}

Die Firma Deutzer Technische Kohle GmbH (DTK) hat sich im In- und Ausland durch innovative Meßtechnik zur Prüfung des Systems Fahrleitung/Schleifleiste/Stromabnehmer und ihre umweltfreundlichen Schleifleisten einen guten Namen erworben. Die DTK-Meßsysteme und Dienstleistungen wurden 1997 in Deutschland, Belgien, Schweden, Österreich und der Schweiz verkauft. Dabei wurden die DTK-Systeme für jeden konkreten Meßauftrag so angepaßt, daß optimale Ergebnisse erreicht werden konnten.

Bei einer der Messungen kam es z. B. darauf an, die Fahrdrahtlage auf der sehr kurzen Kohleschleifleiste über Schienenbogen mit sehr geringem Schienenbogenradius zu ermitteln. Die Untersuchungen erstreckten sich auf unterschiedlich gefederte Fahrzeugtypen und sollten auch eventuelle Unterschiede im System Fahrleitung/Schleifleiste bei einem und bei mehreren anliegenden Stromabnehmern aufzeigen. Als Ergebnis der Messungen konnte ein Unterschied der Fahrleitungslage auf der Schleifleiste bei luftgefederten Fahrzeugen und herkömmlich gefederten Fahrzeugen von bis zu $237 \mathrm{~mm}$ nachgewiesen werden. Diese Unterschiede hängen entscheidend von der Schienenbogenform und der Überhöhung der Schienen zueinander sowie von der Federung des Fahrzeugs ab. Zwischen den Fahrdrahtaufhängungen über einem Schienenbogen sind die Seitenlageunterschiede fuir die verschieden gefederten Fahrzeuge geringer, als direkt unter der Fahrdrahtaufhängung.

Bei mehreren anliegenden Stromabnehmern wird die Fahrleitung stärker angehoben, als bei einem Stromabnehmer. Die seitliche Fahrdrahtlage wird hierdurch beeinflußt. Im Zusammenhang mit der Messauswertung wurden von DTK Empfehlungen erarbeitet, die helfen, das System zu stabilisieren. Vom Auftraggeber wurden besonders diese Empfehlungen geschätzt.

Bei anderen Messungen ging es darum, die Ursachen für das Auftreten von Querrillen und Kantenausbrüchen an der Schleifkohle zu finden. Dabei wurden durch DTK die Orte der Störungen, die dort gefahrene Fahrgeschwindigkeit und die Ursachen für die Stöße zwischen der Fahrleitung und der Schleifleiste ermittelt. Von den Verkehrsunternehmen wurde DTK bescheinigt, daß „im Nachgang der Netzbefahrung eine weitgehende Fehlerbeseitigung erreicht wurde und allgemein die Oberleitungs- und Stromabnehmerschäden drastisch zurückgegangen sind.“

Eine andere Fragestellung ist die Ermittlung der optimalen Einbaulage von Bauteilen der Fahrleitung, wie Strek- kentrenner oder Kreuzungen zwischen Straßenbahnund O-Bus-Fahrleitungen. Für diese Untersuchungen sind die DTK-Systeme Stoß/Zick-Zack hervorragend geeignet. Bei entsprechenden Messungen konnten die Einfluisse der Einbaulage von Streckentrennern auf die Größe des Stoßes ermittelt werden und durch Änderung der Lage der Bauteile konnte die Stoßbelastung im System minimiert werden. Dabei wurde auch nachgewiesen, daß bei der Verwendung von Einholm-Stromabnehmern die Wucht des Stoßes auf die Schleifleiste entscheidend von der Fahrtrichtung des Stromabnehmers abhängt. Der Unterschied kann bis zu $100 \%$ betragen. Durch eine Optimierung des Zick-Zack der Fahrleitung konnte erreicht werden, daß die Kohle über eine maximale Breite ausgenutzt wird. Das fuihrte zu einer Erhöhung der Laufleistung der Schleifleisten und dadurch zur Reduzierung der Kosten.

Bei anderen Untersuchungen sollte das Zusammenwirken des Stromabnehmers mit der Fahrleitung im normalen Linienverkehr geprüft werden. Mit Hilfe des DTK Meßsystems „Fahrdynamik und Fahrdrahthöhe“ wurde nachgewiesen, daß der verwendete Stromabnehmer bei bestimmten Fahrgeschwindigkeiten und Arbeitshöhen erheblich von der vorgegebenen Soll-Anpreßkraft abweicht.

Die Kontaktpreßkraft der zwei Schleifleisten mit der Fahrleitung kann in Abhängigkeit von den Systembedingungen (Beschleunigung, gesamte Anpreßkraft...) sehr stark schwanken. Bei einer Prüfung konnte u. a. festgestellt werden, daß der Stromabnehmer der Fahrdrahthöhenänderung bei der Ausfahrt aus einer Brüicke nicht folgen konnte. Die Anpreßkraft sank auf $24 \%$ der SollAnpreßkraft. Bei der Einfahrt in die Brücke erfolgte die Kontaktierung nur über eine Schleifleiste. Der gesamte Fahrstrom wurde über diese Schleifleiste entnommen. Die Folge sind erhöhter Fahrleitungs- und Schleifleistenverschleiß.

Auch bei der Abnahmeprüfung von neu errichteten Fahrleitungen wurden mit Hilfe der DTK-Systeme Schwachstellen ermittelt, die von der Errichterfirma sofort behoben wurden. Es wurde außerdem geprüft, ob die SollLage mit der Ist-Lage übereinstimmt.

Neu im Programm sind die DTK-Systeme zur Stromabnehmerprüfung. Bei diesen Prüfungen wird der Stromabnehmer mit einer definierten Geschwindigkeit vom höchsten zum niedrigsten und anschließend wieder zum höchsten Arbeitspunkt gefahren. Während der Bewegung wird die Kraft, mit der die Wippe nach oben 
gedrückt wird, bestimmt. Aus der aufgenommenen Hysteresiskurve können Aussagen über die Lagerreibung des Stromabnehmers, die Gleichmäßigkeit der Anpreßkraft usw. abgeleitet werden. Bei der Messung der Seitenstabilität des Stromabnehmers wird die Wippe bei einer mittleren Arbeitshöhe des Stromabnehmers zur Seite (Senkrecht zur Fahrtrichtung) gezogen. Die Zugkraft wird kontinuierlich von $0 \mathrm{kp}$ auf $30 \mathrm{kp}$ erhöht und anschließend auf $0 \mathrm{kp}$ gesenkt. Während dieser Krafteinwirkung wird die seitliche Auslenkung der Wippe bestimmt. Aus der aufgenommenen Hysteresiskurve können Aussagen über die Seitenstabilität des Stromabnehmers abgeleitet werden. Mit Hilfe des Systems Stoß kann getestet werden, welche der Wippenarten auf schwierigen Fahrabschnitten problemlos arbeiten.
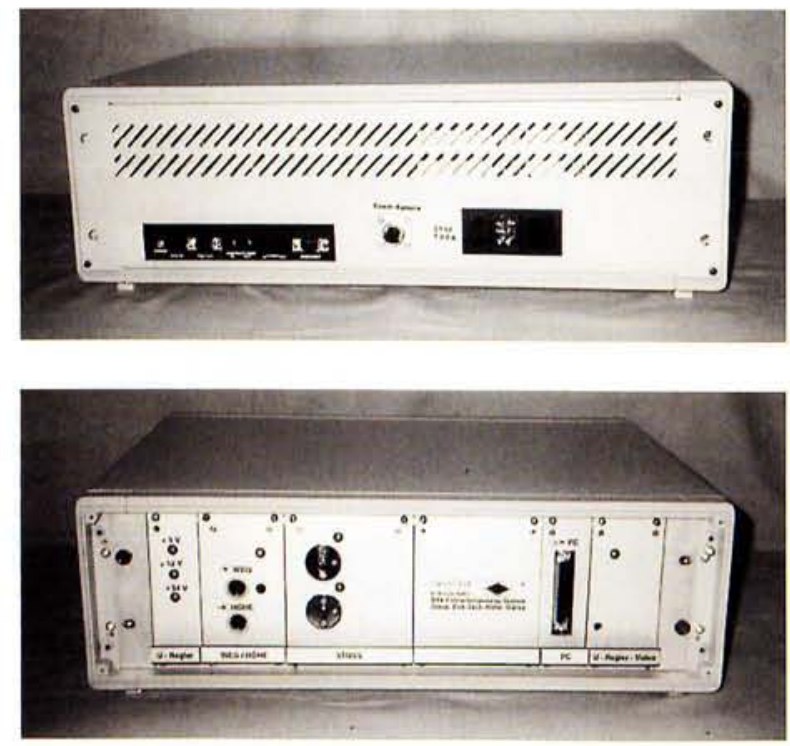

Das Bild zeigt das Herzstück der DTK-Meßsysteme. Durch die 19"-Technik können die für die jeweilige Meßaufgabe erforderlichen Einschübe verwendet werden, so daß das DTK-System für alle Untersuchungen geeignet ist.

Hier ist auch bereits einen Steckplatz für das zur Zeit in Entwicklung befindliche DTK-System „Fahrdrahtstärke“ vorgesehen. Mit diesem neuen System soll die Stärke des Fahrdrahtes während einer Meßfahrt quasi kontinuierlich (d. h. ca. alle $4 \mathrm{~cm}$ bei $30 \mathrm{~km} / \mathrm{h}$ ) gemessen werden. Die ermittelten Extrem- und Mittelwerte werden in kurzen Intervallen auf einem PC dargestellt und zur späteren Auswertung gespeichert. Durch die hohe Meßdichte werden mit geringem Zeitaufwand alle kritischen Verschleißstellen ermittelt. Die Messung erfolgt dabei mit einem Laserscanner in horizontaler Richtung (parallel zur Verschleißfläche). Die so ermittelte Höhe des Fahrdrahtes zeigt im Vergleich zur Höhe eines neuen Fahrdrahtes den Verschleiß an. Die ScannerAnordnung wird in einem speziell geformten Schlitten untergebracht, der durch einen schräggestellten Mast, ähnlich einer O-Bus-Stromabnehmerrute, am Fahrdraht entlanggeführt wird. Bei „Entgleisung“ des Schlittens sorgt eine Schnellabsenkung dafür, daß Kollisionen der Meßeinrichtung mit Oberleitungsbauteilen vermieden werden.
Seit Mitte vorigen Jahres gehören auch komplette Stromabnehmer zum Sortiment von DTK. Diese Stromabnehmer zeichnen sich durch die spezielle U-Bügel-Wippe aus, die für das Zusammenwirken des Stromabnehmers mit der Fahrleitung entwickelt wurde. Die Stromabnehmerwippe kann speziell auf die Fahrleitungsverhältnisse angepaßt werden. Auf Kundenwunsch wurde die U-Bügel-Wippe bereits auf unterschiedliche Stromabnehmertypen aufgebaut.

Auch beim Neuaufbau von defekten Stromabnehmern oder bei der Ersatzteilbereitstellung für Stromabnehmer beschreitet DTK neue Wege.

Im Laufe der Jahre hat DTK viel Erfahrung bei der Optimierung des Systems Fahrleitung - Schleifleiste - Stromabnehmer gewonnen. Unser Know-how kann den Verkehrsbetrieben helfen, ihre Systeme kostengünstiger und wartungsfreundlicher zu gestalten und dabei gleichzeitig einen nicht geringen Beitrag zum Schutz der Umwelt zu leisten.

\section{Verfasser}

\author{
Dipl.-Chem. Manfred Deutzer \\ Geschäftsführer \\ Deutzer Technische Kohle GmbH \\ Bahnhofstraße, 15745 Wildau \\ Tel. (0 33 75) 508-519
}

